\title{
Revascularization by Percutaneous Transluminal Angioplasty for Chronic Mesenteric Ischemia: Results and One-Year Follow-up in a Single Center
}

Miguel Bouzas Cardaci ${ }^{1 *}$, Leslie Remont ${ }^{1}$, Charlotte Ponte ${ }^{1}$, Bernard Van Houte ${ }^{2}$ and Cesar Vazquez ${ }^{1}$

${ }^{1}$ Department of General, Vascular and Thoracic Surgery, CHR Auvelais, Sambreville, Belgium

${ }^{2}$ Department of Gastroenterology, CHR Auvelais, Sambreville, Belgium

\begin{abstract}
Although the prevalence of mesenteric artery stenosis is high, symptomatic chronic mesenteric ischemia (CMI) is rare; the collateral network in the mesenteric circulation serves to prevent most cases of ischemia.

The number of affected vessels is the major determinant in CMI development and most subjects with single vessel mesenteric stenosis do not develop ischemic complaints.
\end{abstract}

Keywords: Angioplasty; Endovascular surgery; Ischemia; Artery stenosis; Abdominal pain; Angiography; Femoral artery

\section{Introduction}

The clinical presentation of CMI consists of postprandial pain, weight loss, and an adapted eating pattern caused by fear of eating. In end-stage disease more continuous pain, diarrhea or a dyspepsia-like presentation can be observed [1].

Both, open surgical revascularization (OR) and endovascular surgery (ES), have been proposed for the treatment of symptomatic CMI. OR was considered the gold standard but ES is being increasingly proposed as the first option.

OR of mesenteric arteries is known for its efficacy and its durability. Sustained improvement in symptoms is noted in $78 \%$ to $100 \%$ of patients, and 3-year patency rates of $76 \%$ to $100 \%$ have been reported. The operative mortality is moderate ( $0 \%$ to $13 \%)$, but post-operative complications can reach $12 \%$ to $45 \%$ [2-4].

ES, consisting of percutaneous transluminal angioplasty and stenting (PTA/stenting), has been first described in 1980 [5] and has been increasingly applied in other vascular beds in order to decrease the morbidity and mortality associated with OR.

Recent series demonstrated that PTA/stenting was associated with lower morbidity and mortality compared with OR and other studies also showed patency rates of $30 \%$ to $90 \%$ at 2 years and 40 to $88 \%$ at 3 years $[3,4]$.

The purpose of this article is to report the results of PTA/stenting in the treatment of patients with CMI in a single center [6-10].

\section{Materials and Methods}

The authors performed a retrospective study in order to evaluate the results of PTA/stenting in patients who presented with symptoms and angiographic findings of CMI. The surgical procedures were performed in patients presenting at least two of the following digestive symptoms: chronic postprandial abdominal pain, weight loss, a fear of food and digestive troubles (diarrhea, nausea, or vomiting) with at least thrombosis or $70 \%$ stenosis in one or more digestive arteries confirmed by pre-operative computed tomography (CT-scan) angiography.

The hospital records of all patients undergoing treatment for CMI on the Service of Vascular and Thoracic Surgery of the CHR Auvelais,
Sambreville, Belgium, were prospectively studied from 2013 to 2016.

Exclusion criteria were patients treated for acute mesenteric ischemia, no atherosclerotic causes of CMI and treatment of any digestive artery in combination with another aortic procedure.

Patient demographics, cardiovascular risk factor (diabetes, hypertension, dyslipidemia, tobacco and alcohol use), comorbid conditions, (cardiac, renal, neurological) and angiographic features were abstracted. The results of follow-up imaging study were recorded and the patency of the revascularization or the earliest date of symptom recurrence or mesenteric vascular disease recurrence was also recorded.

The primary end-points were in-hospital major morbidity and mortality and technical and primary clinical success. In-hospital major morbidity was defined as bowel infarction, myocardial infarction, stroke, acute renal failure, respiratory failure, or multisystem organ failure. Technical success was defined as successful completion of the procedure and $<30 \%$ residual stenosis at the end of the procedure. Primary clinical success was defined as uninterrupted relief or improvement of presenting symptoms with a patent re-vascularized target vessel.

The secondary end-points were death after hospital discharge (overall mortality), symptomatic and radiographic recurrence and reintervention. Radiographic recurrence was the finding of evidence of recurrent stenosis ( $70 \%$ or more) on the vessels previously undergoing treatment by any conventional imaging modality. Symptomatic recurrence was defined as the return of the original symptoms despite intervention.

*Corresponding author: Miguel Bouzas Cardaci, Department of General, Vascular and Thoracic Surgery, CHR Auvelais, Sambreville, Belgium, Tel: +3271265211; E-mail: bouzas_m@hotmail.com

Received December 11, 2017; Accepted December 21, 2017; Published December 27, 2017

Citation: Cardaci MB, Remont L, Ponte C, Houte BV, Vazquez C (2017) Revascularization by Percutaneous Trans luminal Angioplasty for Chronic Mesenteric Ischemia: Results and One-Year Follow-up in a Single Center. J Vasc Med Surg 5: 353. doi: 10.4172/2329-6925.1000353

Copyright: (c) 2017 Cardaci MB, et al. This is an open-access article distributed under the terms of the Creative Commons Attribution License, which permits unrestricted use, distribution, and reproduction in any medium, provided the original author and source are credited. 
Citation: Cardaci MB, Remont L, Ponte C, Houte BV, Vazquez C (2017) Revascularization by Percutaneous Trans luminal Angioplasty for Chronic Mesenteric Ischemia: Results and One-Year Follow-up in a Single Center. J Vasc Med Surg 5: 353. doi: 10.4172/2329-6925.1000353

Page 2 of 3

Clinical and radiographic follow-up was performed with duplex ultrasound (DUS) and CT-scan angiography at one, six and twelve months postoperatively.

\section{Results}

\section{Patients features}

From March 3, 2013, to June 26, 2016, 14 patients underwent ES for $\mathrm{CMI}$ at the CHR Auvelais, Sambreville, Belgium. In the cohort, there were 8 women and 6 men (mean age, 72, 7 years; range, 59 to 85 years).

Traditional cardiovascular risk factors were highly prevalent: hypertension $78.5 \%$ [11], dyslipidemia $64.2 \%$ [9], diabetes $28.5 \%$ [4], nicotine use 50\% [7], and alcohol use $85.7 \%$. Cardiac, renal, and cerebral comorbidities were present in $4(28.5 \%), 2(14.2 \%)$ and 1 (7.1\%) patients, respectively. Patient's characteristics are summarized in Table 1.

Abdominal pain and weight loss was the most common presenting symptom: 14 patients (100\%). Other common symptoms on presentation included diarrhea 50\% [7], fear of eating $21.4 \%$ [3], and nausea $14.2 \%$ [2].

CT-scan was performed in all patients. In our cohort, eight patients (57.1\%) had involvement of one vessel, six (42.9\%) had two-vessel involvement, and 0 had involvement of all three mesenteric arteries with a mean number of 1.33 (Table 2).

\begin{tabular}{|l|c|}
\hline Mean age & 72.7 \\
\hline Female & 8 \\
\hline Male & 6 \\
\hline Cardiovascular risks & $11(78.5 \%)$ \\
\hline Hypertension & $4(28.5 \%)$ \\
\hline Diabetes & $9(64.2 \%)$ \\
\hline Dyslipidemia & $7(50 \%)$ \\
\hline Smoking & $12(85.7 \%)$ \\
\hline Alcohol use & \\
\hline Comorbidities & $4(28.5 \%)$ \\
\hline Cardiac & $2(14.2 \%)$ \\
\hline Renal & $1(7.1 \%)$ \\
\hline Cerebral & \\
\hline
\end{tabular}

Table 1: Demographics and clinical data.

\begin{tabular}{|l|c|}
\hline Vessels involved & \\
\hline SMA & 6 \\
\hline CA & 2 \\
\hline SMA + CA & 6 \\
\hline Total & 20 \\
\hline Vessels treated & 10 \\
\hline SMA & 2 \\
\hline CA & 2 \\
\hline SMA + CA & 16 \\
\hline Total & 1 \\
\hline Type of stent used & 11 \\
\hline 4 mm & 4 \\
\hline 5 mm & 0 \\
\hline 6 mm & $14(100 \%)$ \\
\hline In-hospital mortality/morbidity & $2(14.2 \%)$ \\
\hline Technical and primary clinical success & $1(7.1 \%)$ \\
\hline Overall mortality at 1-years & \\
\hline Recurrence and re-intervention & \\
\hline
\end{tabular}

Table 2: Vessels involved, revascularization and outcomes.

\section{Endovascular techniques}

The ES cohort consisted of 14 patients in whom 16 vessels were re-vascularized.

Access was obtained through the femoral artery in all patients. Selective angiography of the celiac artery (CA) and superior mesenteric artery (SMA) was attempted using a $7 \mathrm{Fr}$ Optitorque angiographic catheter (Terumo Europe NV).

The lesions were crossed using a $0.035^{\prime}$ guidewire $\mathrm{M}$ standard type (Terumo Europe NV) and the stent was deployed at 12 ATM (stents used are summarized in Table 2 followed by post-dilatation if needed. No peri-procedural complications were reported.

A total of 16 vessels were treated, including 12 SMA and 4 CA.

Twelve patients (85.7\%) had one vessel treated, two patients (14.3\%) had two vessels treated and no patients had all three re-vascularized. Revascularizations characteristics are summarized in Table 2.

Post procedurally, all patients were treated with statin and acetylsalicylic acid $80 \mathrm{mg}$ in the first year and clopidogrel $75 \mathrm{mg}$ during the first three months.

\section{Outcomes}

Technical and primary clinical success rates were 100\% with complete resolution of symptoms in all patients at 3 weeks. No immediate failures were reported. In hospital major complications and mortality rates were $0 \%$. The hospital length of stay rate was 3 days [2-6].

Follow-up at 1 year was successful in 12 patients. The overall mortality was $14.2 \%$ (2 patients).

There were two non-post procedural deaths at 6 months: one patient died from myocardial infraction and one died due to suicide.

The recurrence and re-intervention rate was $7.1 \%$ (1 patient). At 10 months after the procedure, one patient showed symptomatic and radiographic recurrence and was retreated by PTA. Angiographic control showed no signs of restenosis but the patient remained symptomatic due, surely, to a secondary gastrointestinal disorder.

The remaining 11 patients (78.5\%) showed no symptoms or recurrence in angiographic follow-ups.

There was no difference in patency, complication, or survival rates irrespective of the number and vessel treated.

\section{Discussion}

OR has been the standard treatment of CMI since the first successful repair reported by Shaw from the Massachusetts General Hospital in 1958 [6]. At present, OR is preferred in lower risk patients and ES is preferred in higher risk patients. This concept is based in some observational studies that prove that ES has lower mortality and morbidity, shorter length of stay and recovery time compared to OR, but more frequent recurrence of symptoms, restenosis and reinterventions. In general, ES is considered as a less durable treatment. However, in those series, patients undergoing ES were older and had more comorbidities than patients undergoing OR. So, more highrisk patients were treated with $\mathrm{PMA} /$ stenting, probably leading to reported outcomes that disadvantage PMA/Stenting [7-9]. In addition, mesenteric revascularization in the stent era (including the recent use of covered stents) might have different results. Some of the patients 
Citation: Cardaci MB, Remont L, Ponte C, Houte BV, Vazquez C (2017) Revascularization by Percutaneous Trans luminal Angioplasty for Chronic Mesenteric Ischemia: Results and One-Year Follow-up in a Single Center. J Vasc Med Surg 5: 353. doi: 10.4172/2329-6925.1000353

Page 3 of 3

in the first series were treated only with angioplasty without stenting $[8,10]$. This leads us to conclude that results of OR and ER are not comparable in some studies.

To our knowledge, no randomized trial exists in the literature comparing OR with PTA and the best available level of evidence is $2 b$. However, recent observational studies with good validity have shown similar patency at medium term follow-up, concluding that ES may be considered a first-line therapy in selected patients. A recent article published in 2016 describing the current insights into diagnosis and treatment of mesenteric ischemia, reach similar conclusions: PMA/ Stenting should be the first choice treatment for CMI. Bypass surgery should only be used in low risk patients who have unfavorable mesenteric lesions, failed PMA/Stenting or Retrograde Open Mesenteric Stenting or multiple recurrences of in-stent stenosis/occlusion [7].

Our study confirmed the low incidence of complications as well as the good results at short term. However, it has several limitations. First, it is a retrospective study performed at a single institution. Second, the study population is small (14 patients) and the study period is short (1 year).

Nevertheless, based on the latest reports and on our initials results, we believe that ES could be the first choice treatment in patients with short stenosis of the involved vessels, in which angioplasty and stent placement would not compromise the landing site for a possible future open bypass graft. OS should still be the preferred approach in patients at low risk for aortic operations who present with complex occlusive disease of their mesenteric vessels (occlusion, long stenosis), or in patients in whom placement of a stent would compromise subsequent bypass grafting [11].

A large randomized study would then be required, but is a difficult task due to the low number of patients potentially suitable for both techniques.

\section{Conclusions}

The authors confirm that PTA/Stenting for patients with CMI is feasible and effective with a low incidence of complications and good results at short term, as others studies had previously concluded.
ES is preferred for high-risk patients and may be an alternative to OR in low-risk patients with ideally suited lesions. However, whether the use of ES in low risk patients should be the standard of practice is yet to be determined.

\section{References}

1. Kolkman JJ, Geelkerken RH (2017) Diagnosis and treatment of chronic mesenteric ischemia: An update. Best Pract Res Clin Gastroenterol 31: 49-57.

2. Zerbib P, Lebuffe G, Sergent-Baudson G, Chamatan A, Massouille D, et al (2008) Endovascular versus open revascularization for chronic mesenteric ischemia: a comparative study. Langenbecks Arch Surg 393: 865-870.

3. Atkins MD, Kwolek CJ, LaMuraglia GM, Brewster DC, Chung TK, et al. (2007) Surgical revascularization versus endovascular therapy for chronic mesenteric ischemia: a comparative experience. J Vasc Surg 45: 1162-1171.

4. Oderich GS, Bower TC, Sullivan TM, Bjarnason H, Cha S, et al. (2009) Open versus endovascular revascularization for chronic mesenteric ischemia: riskstratified outcomes. J Vasc Surg 49: 1472-1479.

5. Furrer J, Grüntzig A, Kugelmeier J, Goebel N (1980) Treatment of abdomina angina with percutaneous dilatation of an arteria mesenterica superior stenosis. Preliminary communication. Cardiovasc Intervent Radiol 3: 43-44.

6. Shaw RS, Maynard EP (1958) Acute and chronic thrombosis of the mesenteric arteries associated with malabsorption; a report of two cases successfully treated by thromboendarterectomy. N Engl J Med 258: 874-878.

7. Blauw JT, Bulut T, Oderich GS, Geelkerken BR, Dutch Mesenteric Ischemia Study Group (2017) Mesenteric vascular treatment 2016: from open surgical repair to endovascular revascularization. Best Pract Res Clin Gastroenterol 31: $75-84$

8. Ke SR, Chiang FT (2010) Endovascular therapy for a patient with chronic mesenteric ischemia. J Formos Med Assoc 109: 315-319.

9. Lejay A, Georg Y, Tartaglia E, Creton O, Lucereau B, et al. (2015) Chronic mesenteric ischemia: 20 year experience of open surgical treatment. Eur J Vasc Endovasc Surg 49: 587-592.

10. Schermerhorn ML, Giles KA, Hamdan AD, Wyers MC, Pomposelli FB (2009) Mesenteric revascularization: management and outcomes in the United States, 1988-2006. J Vasc Surg 50: 341-348.

11. Gupta PK, Horan SM, Turaga KK, Miller WJ, Pipinos II (2010) Chronic mesenteric ischemia: endovascular versus open revascularization. J Endovasc Ther 17: 540-549. 